\title{
Change in Urine Albumin-to-Creatinine Ratio and Risk of Diabetic Peripheral Neuropathy in Type 2 Diabetes: A Retrospective Cohort Study
}

\author{
Ming Zhong ${ }^{1-3}$ \\ Yi-Ru Yang ${ }^{4}$ \\ Yong-Ze Zhang ${ }^{1-3}$ \\ Sun-Jie Yan $^{1-3}$ \\ 'Department of Endocrinology, The First \\ Affiliated Hospital, Fujian Medical \\ University, Fuzhou, 350005, Fujian, \\ People's Republic of China; ${ }^{2}$ Fujian \\ Diabetes Research Institute, The First \\ Affiliated Hospital, Fujian Medical \\ University, Fuzhou, 350005, Fujian, \\ People's Republic of China; ${ }^{3}$ Metabolic \\ Diseases Research Institute, The First \\ Affiliated Hospital, Fujian Medical \\ University, Fuzhou, 350005, Fujian, \\ People's Republic of China; ${ }^{4}$ Department \\ of Ultrasound, The Second Affiliated \\ Hospital, Fujian Medical University, \\ Quanzhou, Fujian, 362000, People's \\ Republic of China
}

Purpose: This study aimed to assess association between change in urine albumin-tocreatinine ratio (UACR) and the risk of diabetic peripheral neuropathy (DPN) in type 2 diabetes mellitus.

Patients and Methods: A retrospective study was performed, which included 185 individuals with type 2 diabetes. At baseline, and at two-year follow-up, we collected basic data, recorded symptoms and signs of DPN, measured biochemical indicators, composite motor nerve conduction velocity (composite $\mathrm{MCV}$ ), and composite sensory nerve conduction velocity (composite SCV).

Results: Changes of composite SCV, MCV and TCSS among different changes in UACR in patients without DPN and with DPN were not significantly different. An increase in UACR $\geq 30 \%$ (OR 3.059, 95\% ; CI: 1.012-9.249) suggested a risk for new-onset DPN. Based on ROC curve analysis, the areas under the curve were $0.654 \pm 0.066$ for change of UACR levels in non-DPN patients.

Conclusion: Change in UACR and NCV was not related in patients without DPN and with DPN; change in UACR $\geq 30 \%$ suggested a risk for new-onset DPN.

Keywords: diabetic peripheral neuropathy, type 2 diabetes, urine albumin-to-creatinine ratio, nerve conduction velocity

\section{Introduction}

As a predictive marker of renal and cardiac outcomes, the urine albumin-tocreatinine ratio (UACR) has been incorporated into the definition and staging of chronic kidney disease. ${ }^{1}$ Reportedly, a high UACR is associated with the risk of left ventricular hypertrophy, ${ }^{2}$ arterial stiffness, carotid atherosclerosis, ${ }^{3}$ diabetic peripheral arterial disease (PAD), ${ }^{4}$ onset of diabetic retinopathy, ${ }^{5}$ chronic renal disease and hypertension. ${ }^{6}$

Diabetic peripheral neuropathy (DPN) can lead to neuropathic pain, which worsens the quality of life. ${ }^{7}$ Many studies confirmed that the level of UACR, which represents one of the risk factors of early $\mathrm{DPN}^{8}{ }^{8}$ was higher in patients with neuropathy as compared those without this condition. ${ }^{9}$ The previous research of our team found that even in the normal range, UACR and the risk of DPN showed a positive correlation. ${ }^{10}$ Furthermore, a study in Taiwanese illustrated that macroalbuminuria indicates onset of neuropathic pain in individuals present with DPN. ${ }^{7}$ Nevertheless, there is no evidence reporting a relationship between UACR in the normal range and DPN.
Correspondence: Sun-jie Yan Department of Endocrinology, The First Affiliated Hospital, Fujian Medical University, 20 Tea Road, Taijiang District, Fuzhou, 350005, Fujian, People's Republic of China

Tel +86-059l-87983333

Fax +86-059I-8798I685

Email fjyansunjie@163.com 
Thus, we have evaluated possible associations between change in the UACR and the risk of DPN in type 2 diabetes with the aim of identifying predictors of newonset DPN.

\section{Patients and Methods}

\section{Study Population}

Type 2 diabetes patients were recruited from the Endocrinology Department at the First Affiliated Hospital of Fujian Medical University between November 5, 2008 and April 18, 2017. The following were exclusion criteria for our study: (i) other types of diabetes, gestational diabetes, and acute complications of diabetes mellitus, including diabetic ketoacidosis and hyperosmolar non-ketotic coma; (ii) micro- or macro-albuminuria (UACR $\geq$ $30 \mathrm{mg} / \mathrm{g}$ ) or end-stage kidney disease or dialytic treatment; (iii) other neuropathic conditions including chronic inflammatory demyelinating polyradiculoneuropathy (CIPD), mono-neuropathy, or conditions caused by vitamin B deficiency and thyroid dysfunction; (iv) other diseases such as Guillain-Barre syndrome, cerebral infarction, complicated by degenerative changes in cervical and lumbar vertebra, severe arteriovenous vascular disease; and (v) other neuronal tissue lesions.

\section{Clinical Measurements}

At study registration, and two years later, an experienced physician collected information, which included demographic characteristics, disease duration, lifestyle, medical history and drug-taking history, and then documented symptoms of somatic neuropathy, including numbness, burning, aching, and dystaxia. The body-mass index (BMI, $\mathrm{kg} / \mathrm{m}^{2}$ ) was determined by dividing the weight (in kilograms) by the square of the height (in meters). A 10 g monofilament, a pin, a tendon hammer and a standard $128 \mathrm{~Hz}$ tuning fork were used to complete the neurological examinations.

\section{Biochemical Measurements}

Serum creatinine (Scr), total cholesterol $(\mathrm{TCH})$, triglyceride levels (TG), high-density lipoprotein cholesterol (HDL-C) and low-density lipoprotein cholesterol (LDLC) levels were measured after a 10 -hour overnight fasting. UACR was determined by dividing the urine albumin level by the urine creatinine level during the routine morning urine collection procedure $(\mathrm{mg} / \mathrm{g})$. The glomerular filtration rate (GFR) was estimated in accord with the following equation: eGFR $(\mathrm{mL} / \mathrm{min})=186 \times[\mathrm{Scr}$ $(\mathrm{mmol} / \mathrm{L}) / 88.41]^{-1.154} \times \mathrm{age}^{-0.203}(\times 0.724$ for female $)$.

\section{Neuropathy Assessment}

Participants were tested by the same experienced physicians using standard procedure. Electromyography (EMG) (key point, Alpine Biomed ApS, Denmark) was used to assess motor nerve conduction velocity (MCV) and sensory nerve conduction velocity (SCV). No sources of interference were available in the examination room, and a quiet environment was maintained. The examination room temperature was maintained within a range of $18-25^{\circ} \mathrm{C}$, and local skin temperature was kept constant $\left(28-30^{\circ} \mathrm{C}\right)$. The normal reference values were based on the results of an epidemiological survey conducted in the Chinese population in 1984 by Tang et al. ${ }^{11}$ The threshold for the slowed nerve conduction velocity (NCV) was set at $<20 \%$ of the control nerve conduction velocity. Nerve conduction was considered abnormal if two or more nerves had tested as abnormal. NCV composite $Z$ score was used to evaluate neurological function. Composite $Z$ score calculation method: Composite MCV $Z$ score $($ composite $\mathrm{MCV})=($ median $\mathrm{MCV} Z$ score + ulnar $\mathrm{MCV}$ $Z$ score + peroneus MCV $Z$ score + tibial MCV $Z$ score $) / 4$. Composite SCV $Z$ score $($ composite SCV $)=($ median SCV $Z$ score + ulnar SCV $Z$ score + peroneal SCV $Z$ score + sural $\mathrm{SCV} Z$ score $) / 4$. Among them, $\mathrm{NCV} Z$ score $=$ (measured value of each patient-mean value of normal control group)/standard deviation (SD) of normal control group. $^{12}$

\section{Diagnostic Criteria}

(i) The criteria for type 2 diabetes was based on the criteria established by the World Health Organization in 1999. (ii) A diagnosis of hypertension was defined according to the JNC 7 report. $^{13}$ (iii) In accordance with the criteria proposed by an International European and North American Expert Committee, ${ }^{14}$ DPN was defined as those patients that presented with diabetes who exhibited abnormal nerve conduction velocity, including diagnosis and subclinical DPN (DPN was confirmed as abnormal nerve conductions if there were no signs or symptomology of neuropathy). (iv) a change rate of UACR was estimated by the following equation: $100 \% \times$ [Second UACR $(\mathrm{mg} / \mathrm{g})])$ - Baseline UACR $(\mathrm{mg} /$ $\mathrm{g})] /$ Baseline UACR $(\mathrm{mg} / \mathrm{g})$. According to prior research, ${ }^{14}$ Decrease group was defined as follows: change rate of UACR $\leq-30 \%$; Minor group, |change 
rate of $\mathrm{UACR} \mid<30 \%$; Increase group, change rate of $\mathrm{UACR} \geq 30 \%$.

\section{Statistical Analysis}

Skewed data was expressed as the median across the 25 th and 75th quartiles, while normally distributed data was expressed as the mean \pm standard deviation (SD). Differences at baseline were analyzed by analysis of variance (ANOVA) or the rank test for continuous variables or the Chi-squared test for categorical variables. Data at baseline and follow-up were compared with the paired Student's $t$-test. Independent factors for DPN were identified by multivariate logistic regression analysis. Dual logistical regression analysis was performed to examine the association among altered UACR values, onset of DPN, and nerve conduction velocity. The receiver operating characteristic curve (ROC) was used to identify the cut-off of UACR values, which indicated DPN. Statistical analyses were performed with SPSS version 19.0 statistical software. A two-sided $P$-value $<0.05$ was considered statistically significant.

\section{Results}

\section{Study Population Characteristics}

In this study, 185 patients were enrolled. There were 116 patients without DPN (mean age $59.33 \pm 10.92$; male/ female ratio, 55/61, with a mean diabetic duration of $8.12 \pm 7.94$ years), and 69 patients with DPN (mean age $60.32 \pm 9.28$; male/female ratio, $37 / 32$, with a mean diabetic duration of $9.81 \pm 6.58$ years).

Significant differences were observed in the baseline composite SCV and the follow-up composite MCV among the three groups of patients that did not present with DPN $(P<0.05)$, while in patients with DPN, the change in UACR gave a negative correlation to the baseline composite $\mathrm{MCV}$ $(P=0.71$; Tables 1 and 2). In patients without DPN, 31 participants experienced the outcome of incident DPN (Supplementary Table 1). In DPN patients, 18 participants experienced SCV deterioration and nine participants experienced MCV deterioration (Supplementary Tables 2 and $\underline{3}$ ).

\section{Changes in NCV and TCSS}

In patients without DPN, changes of composite SCV and $\mathrm{MCV}$ among three groups were not significantly different (Figure 1A and B). There was no significant difference in change of Toronto clinical scoring system (TCSS) between three groups (Figure 1C).
In DPN patients, there were no statistical difference in changes of composite SCV and MCV (Figure 2A and B). Change of TCSS was not statistically different between three groups (Figure 2C).

\section{Onset and Prognosis of DPN}

In patients without DPN, after adjusting for confounding factors that included the age of the patient, gender, diabetic duration, HbA1C, history of hypertension, use of hypoglycemic drugs, angiotensin converting enzyme inhibitor (ACEI), and statins, increased in UACR $\geq 30 \%$ suggested a risk for new-onset DPN (OR 3.059, 95\% CI: 1.012-9.249) (Figure 3). In patients with DPN, UACR change was not a risk factor for change in NCV (Figure 4).

\section{ROC Analysis to Indicate DPN}

Based on receiver operating characteristic (ROC) analysis, the areas under the curve (AUC) were $0.654 \pm 0.066$ UACR change in patients without DPN (Figure 5).

\section{Discussion}

In our study, change of composite SCV, MCV and TCSS among different changes of UACR in patients without DPN and with DPN were not significantly different. But change of UACR $\geq 30 \%$ suggested a risk for new-onset DPN. In patients with DPN, change of UACR was not a risk factor for new DPN.

At present, the relationship between altered UACR and neuropathy remains unclear, while other important complications that include kidney disease were shown to be positively correlated with urinary protein levels. A study on 56,946 United States veterans confirmed that changes in albuminuria were linearly associated with a risk of kidney outcomes over a 1-year interval. ${ }^{15}$ Moreover, Viazzi et al also found that persistent albuminuria was associated with a greater risk of kidney events, and changes in albuminuria were associated with a paralleled risk of renal disease. ${ }^{16}$ Moreover, in a 2-year cohort study, patients that demonstrated exacerbations from normoalbuminuria to microalbuminuria were demonstrated to have a higher risk than those exhibited an improvement from a state of microalbuminuria to normoalbuminuria. ${ }^{17}$ A cross-sectional study confirmed that UACR was a risk factor for peripheral neuropathy. ${ }^{8}$ This study showed that diabetic neuropathy was associated with DPN but lacked follow-up. Our study first demonstrated that an increase in UACR was also a risk of DPN in non-DPN patients, which complemented the above studies and may 
Table I Study Population Characteristics of Non-DPN

\begin{tabular}{|c|c|c|c|c|}
\hline & \multicolumn{3}{|c|}{ UACR Change Over Two Years } & \multirow[b]{2}{*}{$P$} \\
\hline & Decrease Group & Minor Group & Increase Group & \\
\hline Age (years) & $57.45 \pm 10.75$ & $58.29 \pm 11.47$ & $62.15 \pm 10.53$ & 0.15 \\
\hline Female [n (\%)] & I8 (54.5\%) & $17(4 \mid .5 \%)$ & $24(61.5 \%)$ & 0.19 \\
\hline Diabetes duration (years) & $7.46 \pm 6.29$ & $6.46 \pm 5.65$ & $9.52 \pm 9.64$ & 0.18 \\
\hline Blood glucose-lowering treatments [n (\%)] & 30 (91.0\%) & 37 (90.0\%) & 35 (90.0\%) & 0.99 \\
\hline History of hypertension [n (\%)] & I8 (54.5\%) & $20(48.8 \%)$ & $17(43.6 \%)$ & 0.65 \\
\hline $\mathrm{SBP}(\mathrm{mmHg})$ & $|32.58 \pm 16.7|$ & $130.49 \pm 14.10$ & $130.92 \pm 14.06$ & 0.82 \\
\hline $\mathrm{DBP}(\mathrm{mmHg})$ & $80.06 \pm 10.23$ & $78.12 \pm 9.52$ & $73.21 \pm 10.20$ & $0.01 *$ \\
\hline Heart rate (bpm) & $78.33 \pm 10.00$ & $76.95 \pm 13.37$ & $76.15 \pm 11.37$ & 0.73 \\
\hline QT (ms) & $0.37 \pm 0.03$ & $0.38 \pm 0.02$ & $0.38 \pm 0.03$ & 0.40 \\
\hline QTC (ms) & $0.40 \pm 0.03$ & $0.40 \pm 0.02$ & $0.40 \pm 0.02$ & 0.49 \\
\hline BMI (kg/m2) & $25.37 \pm 2.33$ & $26.07 \pm 3.87$ & $24.87 \pm 3.02$ & 0.25 \\
\hline Scr (umol/L) & $59.49 \pm 15.69$ & $64.52 \pm 14.38$ & $61.45 \pm 22.07$ & 0.47 \\
\hline BUN (mmol/L) & $5.33 \pm 1.63$ & $5.29 \pm 1.53$ & $5.27 \pm 1.50$ & 0.99 \\
\hline $\mathrm{TC}(\mathrm{mmol} / \mathrm{L})$ & $4.76 \pm 1.09$ & $4.75 \pm 1.28$ & $4.52 \pm 0.94$ & 0.58 \\
\hline TG (mmol/L) & $1.98 \pm 2.23$ & $2.45 \pm 2.01$ & $1.36 \pm 0.88$ & $0.03 *$ \\
\hline $\mathrm{HDL}(\mathrm{mmol} / \mathrm{L})$ & $1.18 \pm 0.41$ & $1.09 \pm 0.39$ & $1.29 \pm 0.39$ & 0.10 \\
\hline LDL (mmol/L) & $2.92 \pm 0.92$ & $2.86 \pm 1.14$ & $2.68 \pm 0.91$ & 0.54 \\
\hline vLDL (mmol/L) & $0.87 \pm 1.03$ & $1.07 \pm 0.92$ & $0.60 \pm 0.41$ & $0.04 *$ \\
\hline HbAlc (\%) & $8.34 \pm 2.01$ & $8.36 \pm 2.35$ & $8.08 \pm 2.12$ & 0.82 \\
\hline First UACR(mg/g) & $12.95(8.33,16.95)$ & $6.69(3.90,11.59)$ & $7.66(4.15,18.70)$ & $<0.01 *$ \\
\hline Second UACR (mg/g) & $5.21(4.00,9.19)$ & $5.39(4.13,10.88)$ & $18.55(8.04,69.46)$ & $<0.01 *$ \\
\hline First eGFR (mL/min) & $101.27(95.35,109.89)$ & I0I.II (92.9I,108.34) & 98.91 (90.72,108.37) & 0.30 \\
\hline Second eGFR (mL/min) & $101.73(91.21,|08.5|)$ & $98.77(89.25,107.35)$ & $93.55(86.84,106.15)$ & 0.17 \\
\hline First composite MCV & $-3.07 \pm 0.79$ & $-3.10 \pm 0.79$ & $-3.38 \pm 0.80$ & 0.16 \\
\hline Second composite MCV & $-3.10 \pm 0.86$ & $-2.99 \pm 0.82$ & $-3.48 \pm 0.81$ & $0.03^{*}$ \\
\hline First composite SCV & $-0.38 \pm 0.69$ & $-0.27 \pm 0.78$ & $-1.03 \pm 1.78$ & $0.0 I^{*}$ \\
\hline Second composite SCV & $-0.34 \pm 0.66$ & $-0.26 \pm 0.75$ & $-0.68 \pm 1.00$ & 0.06 \\
\hline First TCSS & $1.31 \pm 2.22$ & $1.83 \pm 2.66$ & $2.41 \pm 2.84$ & 0.22 \\
\hline Second TCSS & $2.73 \pm 3.17$ & $2.63 \pm 3.13$ & $2.71 \pm 3.29$ & 0.99 \\
\hline
\end{tabular}

Notes: Decrease group, change rate of UACR $\leq-30 \%$; Minor group, |change rate of UACR $\mid<30 \%$; Increase group, change rate of UACR $\geq 30 \%$; *was considered statistically significant.

Abbreviations: Composite MCV, composite MCV Z score; composite SCV, composite SCV Z score; DPN, diabetic peripheral neuropathy; GFR, glomerular filtration rate; $\mathrm{HbAlc}$, hemoglobin AIc; HDL-C, high-density lipoprotein cholesterol; LDL-C, low-density lipoprotein cholesterol; MCV, motor nerve conduction velocity; NCV, nerve conduction velocity; ROC, receiver operating characteristic curve; Scr, Serum creatinine; SCV, sensory nerve conduction velocity; TCH, total cholesterol; TCSS, Toronto clinical scoring system; TG, triglyceride levels; UACR, urine albumin-to-creatinine ratio. 
Table 2 Study Population Characteristics of DPN

\begin{tabular}{|c|c|c|c|c|}
\hline & \multicolumn{3}{|c|}{ UACR Change Over Two Years } & \multirow[b]{2}{*}{$P$} \\
\hline & Decrease Group & Minor Group & Increase Group & \\
\hline Age (years) & $58.56 \pm 8.46$ & $59.87 \pm 7.57$ & $61.13 \pm 10.63$ & 0.65 \\
\hline Female [n (\%)] & $9(0.5)$ & $7(0.467)$ & $14(0.452)$ & 0.95 \\
\hline Diabetes duration (years) & $8.00 \pm 4.99$ & $7.50 \pm 4.58$ & $11.94 \pm 7.62$ & $0.04 *$ \\
\hline Blood glucose-lowering treatments [n (\%)] & $15(83.3 \%)$ & $13(86.7 \%)$ & $31(100 \%)$ & 0.07 \\
\hline History of hypertension [n (\%)] & $5(27.8 \%)$ & $8(53.3 \%)$ & 19 (61.3\%) & 0.07 \\
\hline SBP $(\mathrm{mmHg})$ & $131.22 \pm 11.82$ & $134.27 \pm 14.82$ & $135.10 \pm 15.83$ & 0.66 \\
\hline $\mathrm{DBP}(\mathrm{mmHg})$ & $73.56 \pm 9.19$ & $72.93 \pm 4.79$ & $75.65 \pm 8.83$ & 0.50 \\
\hline Heart rate(bpm) & $80.83 \pm 11.06$ & $77.67 \pm 10.04$ & $78.87 \pm 9.19$ & 0.65 \\
\hline $\mathrm{QT}(\mathrm{ms})$ & $0.37 \pm 0.03$ & $0.39 \pm 0.07$ & $0.37 \pm 0.02$ & 0.46 \\
\hline QTC (ms) & $0.41 \pm 0.02$ & $0.39 \pm 0.03$ & $0.41 \pm 0.02$ & 0.15 \\
\hline BMI $\left(\mathrm{kg} / \mathrm{m}^{2}\right)$ & $23.40 \pm 2.49$ & $24.05 \pm 3.57$ & $25.43 \pm 3.79$ & 0.13 \\
\hline Scr (umol/L) & $51.06 \pm 8.62$ & $58.77 \pm 21.38$ & $63.09 \pm 17.58$ & $0.04 *$ \\
\hline BUN (mmol/L) & $5.25 \pm 1.61$ & $5.18 \pm 1.25$ & $5.81 \pm 1.98$ & 0.40 \\
\hline $\mathrm{TC}(\mathrm{mmol} / \mathrm{L})$ & $4.66 \pm 0.90$ & $4.55 \pm 1.01$ & $4.30 \pm 1.00$ & 0.43 \\
\hline TG (mmol/L) & $1.47 \pm 0.57$ & $1.68 \pm 0.83$ & $1.50 \pm 1.02$ & 0.77 \\
\hline $\mathrm{HDL}(\mathrm{mmol} / \mathrm{L})$ & $1.11 \pm 0.32$ & $1.11 \pm 0.31$ & $1.16 \pm 0.28$ & 0.77 \\
\hline LDL (mmol/L) & $2.87 \pm 0.87$ & $2.88 \pm 0.82$ & $2.57 \pm 0.82$ & 0.36 \\
\hline vLDL (mmol/L) & $0.60 \pm 0.32$ & $0.67 \pm 0.40$ & $0.68 \pm 0.47$ & 0.82 \\
\hline HbAlc (\%) & $9.61 \pm 2.08$ & $9.46 \pm 2.66$ & $8.65 \pm 1.78$ & 0.25 \\
\hline First UACR (mg/g) & $9.47(7.33,16.08)$ & $7.39(6.13,11.30)$ & $9.83(4.98,13.23)$ & 0.52 \\
\hline Second UACR (mg/g) & $4.89(3.84,6.68)$ & $7.15(6.17,11.63)$ & $22.47(8.29,47.74)$ & $<0.01 *$ \\
\hline First eGFR (mL/min) & $109.69(101.94,111.13)$ & $104.10(94.87,109.92)$ & $99.60(90.92,107.79)$ & 0.14 \\
\hline Second eGFR (mL/min) & $100.45(92.61,111.44)$ & $101.12(93.69,109.47)$ & $100.35(88.69,108.31)$ & 0.59 \\
\hline First composite MCV Z score & $-3.97 \pm 0.83$ & $-3.80 \pm 0.70$ & $-3.79 \pm 0.75$ & 0.71 \\
\hline Second composite MCV Z score & $-3.58 \pm 1.01$ & $-3.61 \pm 0.87$ & $-3.61 \pm 0.92$ & 0.99 \\
\hline First composite SCV Z score & $-1.65 \pm 0.87$ & $-1.89 \pm 1.31$ & $-1.20 \pm 0.82$ & 0.12 \\
\hline Second composite SCV Z score & $-1.42 \pm 1.27$ & $-1.31 \pm 1.69$ & $-1.24 \pm 1.16$ & 0.90 \\
\hline First TCSS & $5.13 \pm 3.23$ & $4.73 \pm 3.65$ & $3.03 \pm 3.62$ & 0.12 \\
\hline Second TCSS & $3.06 \pm 2.92$ & $4.00 \pm 3.30$ & $3.55 \pm 2.54$ & 0.63 \\
\hline
\end{tabular}

Notes: Decrease group, change rate of UACR $\leq-30 \%$; Minor group, |change rate of UACR $\mid<30 \%$; Increase group, change rate of UACR $\geq 30 \%$; *was considered statistically significant.

Abbreviations: Composite MCV, composite MCV Z score; composite SCV, composite SCV Z score; BMI, body-mass index; DPN, diabetic peripheral neuropathy; GFR, glomerular filtration rate; HbAIc, Hemoglobin A Ic; HDL-C, high-density lipoprotein cholesterol; LDL-C, low-density lipoprotein cholesterol; MCV, motor nerve conduction velocity; NCV, nerve conduction velocity; ROC, receiver operating characteristic curve; Scr, Serum creatinine; SCV, sensory nerve conduction velocity; TC, total cholesterol; TCSS, Toronto clinical scoring system; TG, triglyceride levels; UACR, urine albumin-to-creatinine ratio. 
A

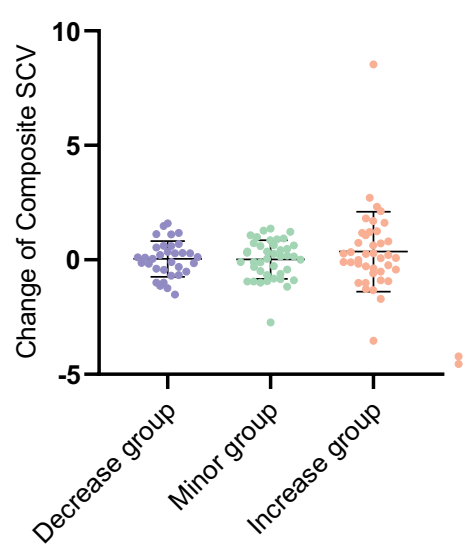

B

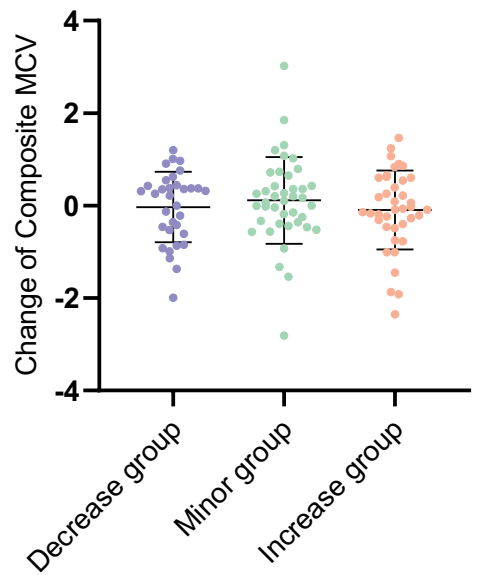

C

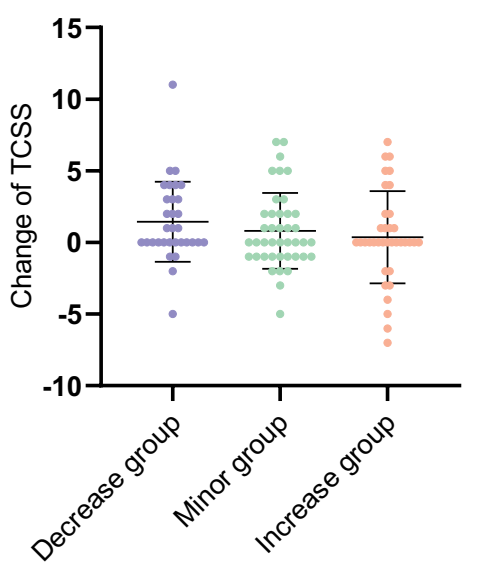

Figure I Changes in NCV and TCSS in non-DPN. (A) Changes of composite SCV in non-DPN; (B) changes of composite MCV in non-DPN; (C) changes of TCSS in nonDPN.

Notes: Decrease group, change rate of UACR $\leq-30 \%$; Minor group, $\mid$ change rate of $U A C R \mid<30 \%$; Increase group, change rate of UACR $\geq 30 \%$.

Abbreviations: Composite MCV, composite MCV Z score; Composite SCV, composite SCV Z score; NCV, nerve conduction velocity; TCSS, Toronto clinical scoring system; non-DPN, type 2 diabetes patient without diabetic peripheral neuropathy; UACR, urine albumin-to-creatinine ratio.

A

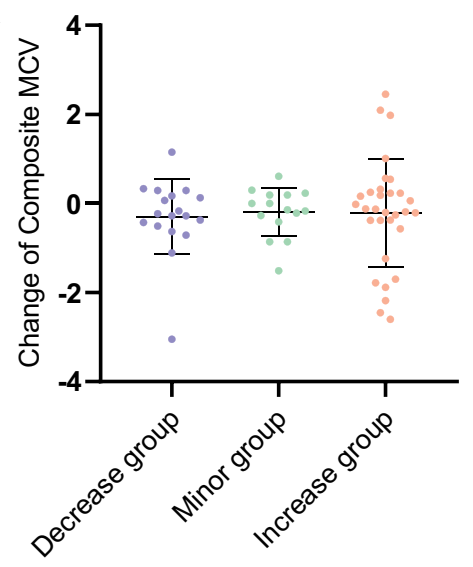

B

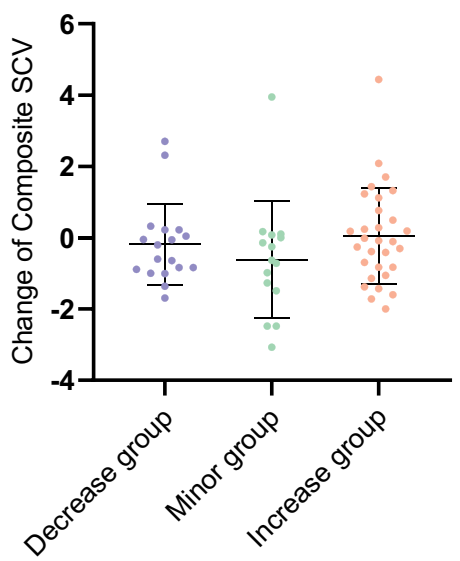

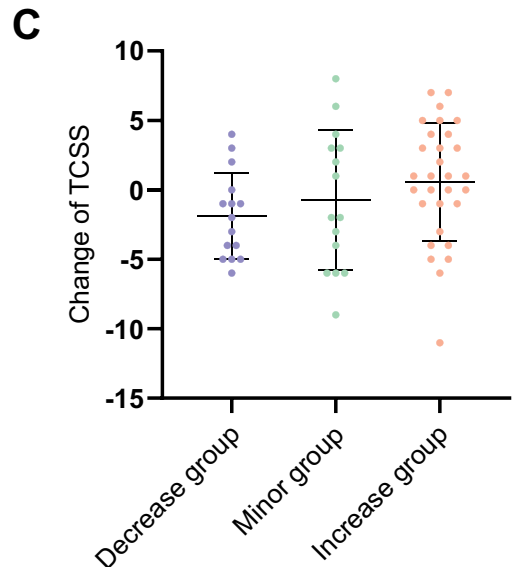

Figure 2 Changes in NCV and TCSS in DPN. (A) Changes of composite SCV in DPN; (B) changes of composite MCV in DPN; (C) changes of TCSS in DPN. Notes: Decrease group, change rate of UACR $\leq-30 \%$; Minor group, $\mid$ change rate of $U A C R \mid<30 \%$; Increase group, change rate of $U A C R \geq 30 \%$. Abbreviations: Composite MCV, composite MCV Z score; Composite SCV, composite SCV Z score; NCV, nerve conduction velocity; TCSS, Toronto clinical scoring system; DPN, type 2 diabetes patient with diabetic peripheral neuropathy; UACR, urine albumin-to-creatinine ratio.

provide clues for further clinical exploration of the relationship between UACR and neuropathy in type 2 diabetes.

Currently, the relationship between a decline in UACR and diabetes-related complications remains controversial. On one hand, Selvaraj et al demonstrated that a decline in UACR improved cardiovascular mortality and all-cause mortality. ${ }^{18}$ In addition, Carrero et al found a linear correlation between the degree of UACR and end-stage renal disease (ESRD), which means that a decrease in UACR might ameliorate ESRD risk. ${ }^{19}$ By contrast, their study indicated that elevated UACR is a risk factor for all-cause mortality, while there was no significant difference between reduced UACR and changes in all-cause mortality. ${ }^{19}$
Our research has some value for guiding clinical practice. Drugs decreasing UACR may reduce the risk of newonset DPN. This kind of research is worthy of further exploration. Furthermore, it is meaningful to monitor UACR more frequently in clinical practice. Once UACR changes, more active intervention should be taken to prevent possible new-onset DPN.

Our study results showed no correlation between change of UACR and NCV change in non-DPN patients and DPN patients. Possible reasons for this include the following: (i) In our study, we enrolled patients with a normal range of UACR that had a relatively better robust of disease; (ii) DPN patients are often complicated with diabetic nephropathy and other 


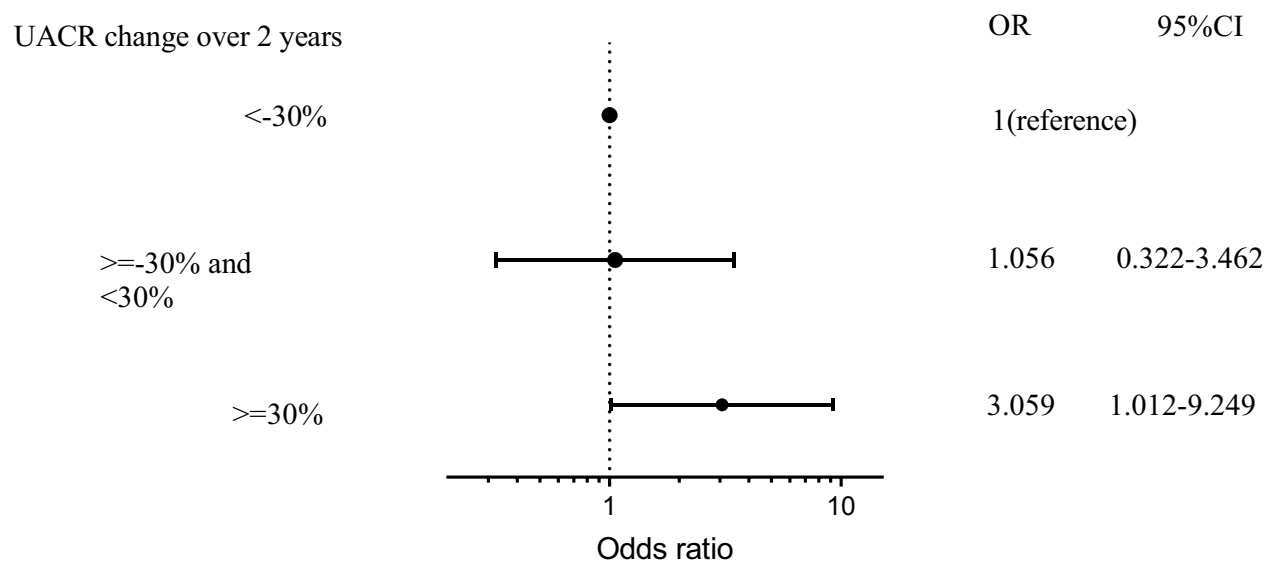

Figure 3 Risk factor for new-onset DPN in non-DPN.

Notes: After adjusting for confounding factors that included the age of the patient, gender, diabetic duration, HbAIC, history of hypertension, use of hypoglycemic drugs, angiotensin converting enzyme inhibitor (ACEI), and statins.

Abbreviations: non-DPN, type 2 diabetes patient without diabetic peripheral neuropathy; UACR, urine albumin-to-creatinine ratio; DPN, type 2 diabetes patient with diabetic peripheral neuropathy; UACR, urine albumin-to-creatinine ratio.

A

UACR change over 2 years

$$
\begin{array}{r}
<-30 \% \\
+<30 \% \\
>=30 \%
\end{array}
$$

B

UACR change over 2 years

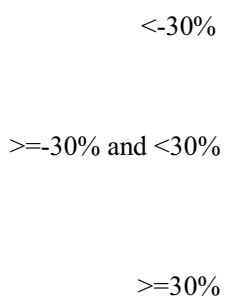

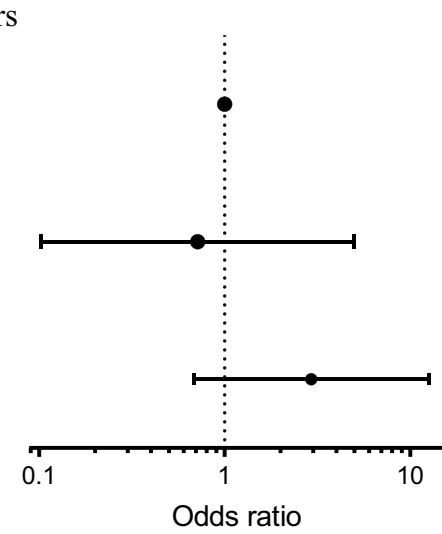

Odds ratio
OR $\quad 95 \% \mathrm{CI}$

1(reference)

$0.715 \quad 0.102-5.009$

$2.928 \quad 0.682-12.571$

OR $\quad 95 \% \mathrm{CI}$

1(reference)

$1.148 \quad 0.065-20.22$

$3.867 \quad 0.418-35.729$

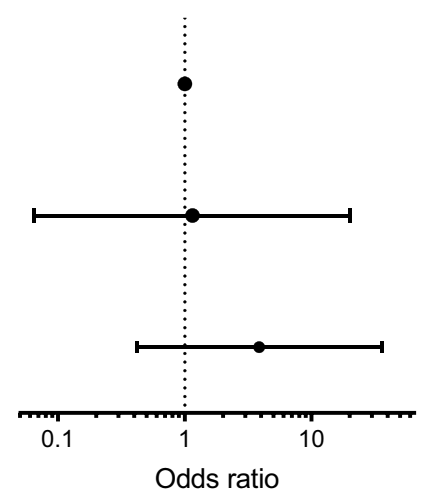

Figure 4 Risk factor for change in NCV in DPN. (A) Risk factor for change in composite SCV in DPN; (B) risk factor for change in composite MCV in non-DPN.

Notes: After adjusting for confounding factors that included the age of the patient, gender, diabetic duration, HbAIC, history of hypertension, use of hypoglycemic drugs, angiotensin converting enzyme inhibitor (ACEI), and statins.

Abbreviations: DPN, type 2 diabetes patient with diabetic peripheral neuropathy; UACR, urine albumin-to-creatinine ratio; Composite MCV, composite MCV Z score; Composite SCV, composite SCV Z score; DPN, type 2 diabetes patient with diabetic peripheral neuropathy; UACR, urine albumin-to-creatinine ratio. 


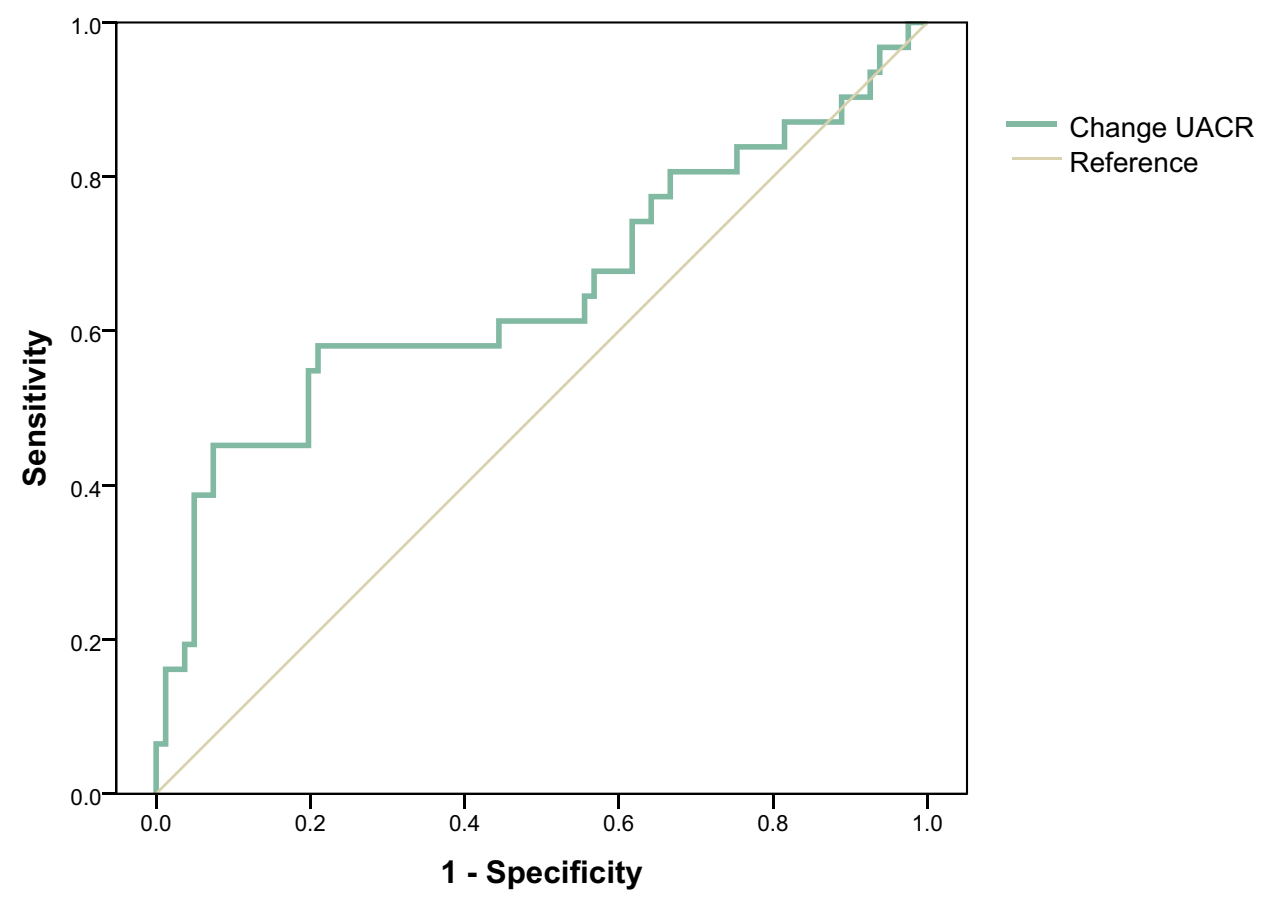

Figure 5 ROC analysis of UACR change in non-DPN patient to indicate DPN.

Abbreviations: non-DPN, type 2 diabetes patient without diabetic peripheral neuropathy; DPN, type 2 diabetes patient with diabetic peripheral neuropathy; ROC, receiver operating characteristic curve.

complications, ${ }^{20,21}$ which might influence UACR; ${ }^{22}$ (iii) compared with non-DPN patients, those patients with DPN might have differences in their medical history such as ACEI and statins that can lead to improved endothelial function and inflammation that can affect urinary protein levels; (iv) under conditions of current medical condition, diabetic peripheral neuropathy is irreversible, and consequently it is difficult to improve nerve conduction velocity; (v) The sample size of this study is relatively small. Collectively, these reasons might have accounted for insignificant difference between observed changes in UACR levels and NCV.

Some limitations of our study should also be mentioned here. First, we performed a single-center study, and thus the universality of the results remains to be evaluated in larger cohort studies. If multi-center and larger samples research can be conducted in the future, it will be more meaningful. Second, our investigation was a retrospective analysis, and there may be selection and loss to follow-up bias. In the future, studies such as drug intervention will help to further confirm the predictive value of UACR changes for newonset DPN. Third, UACR was measured only once per follow-up, and measurement errors were possible. If possible, repeated measurements on UACR can reduce the impact of measurement errors on the results.

\section{Conclusion}

Our study demonstrated the relationship between change of UACR and neuropathy. Though changes in UACR and NCV were not related in patients without DPN and with DPN, change of UACR $\geq 30 \%$ suggested a risk for new-onset DPN. Thus, regularly measuring UACR in non-DPN patients may serve as a monitoring approach for the occurrence of neuropathy.

\section{Abbreviations}

AUC, areas under the curve; BMI, body-mass index; DPN, diabetic peripheral neuropathy; GFR, glomerular filtration rate; HDL-C, high-density lipoprotein cholesterol; LDL-C, low-density lipoprotein cholesterol; $\mathrm{MCV}$, motor nerve conduction velocity; NCV, nerve conduction velocity; ROC, receiver operating characteristic curve; Scr, Serum creatinine; $\mathrm{SCV}$, sensory nerve conduction velocity; $\mathrm{TCH}$, total cholesterol; TCSS, Toronto clinical scoring system; TG, triglyceride levels; UACR, urine albumin-tocreatinine ratio.

\section{Data Sharing Statement}

All data generated or analyzed during this study are included in this published article. 


\section{Ethics Approval and Informed Consent}

This study was conducted in accordance with the Declaration of Helsinki and approved by the Ethics Committee of First Affiliated Hospital of Fujian Medical University (MTCA, ECFAH of FMU [2017]131). Written informed consent was obtained from all participants.

\section{Acknowledgments}

Ming Zhong and Yi-Ru Yang are joint co-first authors.

\section{Funding}

This study was supported by Startup Fund for scientific research, Fujian Medical University (Grant number: 2018QH1077) and the Central Government Special Funds for Local Science and Technology Development (2018L3007).

\section{Disclosure}

No potential conflicts of interest was reported by the author(s).

\section{References}

1. Andrassy KM. Comments on 'KDIGO 2012 clinical practice guideline for the evaluation and management of chronic kidney disease'. Kidney Int. 2013;84(3):622-623. doi:10.1038/ki.2013.243

2. Feng L, Khan AH, Jehan I, Allen J, Jafar TH. Albuminuria and kidney function as prognostic marker of left ventricular mass among South Asians with hypertension. J Am Soc Hypertens. 2017;11(12):811-822. doi:10.1016/j.jash.2017.10.003

3. Yoon HE, Kim ES, Mo EY, Shin SJ, Moon SD, Han JH. High normal albuminuria is associated with arterial stiffness and carotid atherosclerosis in Korean patients with type 2 diabetes. Nutr Metab Cardiovasc Dis. 2015;25(8):787-794. doi:10.1016/j.numecd.2015.03.011

4. Xu B, Dai M, Li M, et al. Low-grade albuminuria is associated with peripheral artery disease in Chinese diabetic patients. Atherosclerosis. 2014;232(2):285-288. doi:10.1016/j.atherosclerosis.2013.11.046

5. Hsieh YT, Tsai MJ, Tu ST, Hsieh MC. Association of abnormal renal profiles and proliferative diabetic retinopathy and diabetic macular edema in an Asian population with type 2 diabetes. JAMA Ophthalmol. 2018;136(1):68-74. doi:10.1001/ jamaophthalmol.2017.5202

6. Ashitani A, Ueno T, Nakashima A, Doi S, Yamane K, Masaki T. Highnormal albuminuria and incident chronic kidney disease in a male nondiabetic population. Clin Exp Nephrol. 2017;22(4):835-842. doi:10.1007/s10157-017-1522-6

7. Pai YW, Lin CH, Lee IT, Chang MH. Prevalence and biochemical risk factors of diabetic peripheral neuropathy with or without neuropathic pain in Taiwanese adults with type 2 diabetes mellitus. Diabetes Metab Syndr. 2018;12(2):111-116. doi:10.1016/j.dsx.2017.09.013
8. Naqvi IH, Talib A, Akhter ST, Abdi SR, Rizvi SNZ, Ubaid M Peripheral neuropathy and vasculopathy; frequency and associated risk factors in newly diagnosed treatment naive type 2 diabetes. Open $J$ Endocr Metab Dis. 2018;08(05):125-136. doi:10.4236/ ojemd.2018.85013

9. Lu B, Yang Z, Wang M, et al. High prevalence of diabetic neuropathy in population-based patients diagnosed with type 2 diabetes in the Shanghai downtown. Diabetes Res Clin Pract. 2010;88(3):289-294. doi:10.1016/j.diabres.2010.02.002

10. Zhang Y, Jiang Y, Shen X, Yan S. Can both normal and mildly abnormal albuminuria and glomerular filtration rate be a danger signal for diabetic peripheral neuropathy in type 2 diabetes mellitus? Neurol Sci. 2017;38(8):1381-1390. doi:10.1007/s10072-017-2946-1

11. Tang XF, Yang T, Yang BX, Liu XZ, Rong ZP. Electromyographic findings in normal Chinese. Analysis of 310 subjects. Chin Med J. 1984;97(8):613-622.

12. Li L, Liu B, Lu J, et al. Serum albumin is associated with peripheral nerve function in patients with type 2 diabetes. Endocrine. 2015;50 (2):397-404. doi:10.1007/s12020-015-0588-8

13. Chobanian AV, Bakris GL, Black HR, et al. The seventh report of the joint national committee on prevention, detection, evaluation, and treatment of high blood pressure: the JNC 7 report. JAMA. 2003;289(19):2560-2571. doi:10.1001/jama.289.19.2560

14. Heerspink HJ, Kropelin TF, Hoekman J, de Zeeuw D. Drug-induced reduction in albuminuria is associated with subsequent renoprotection: a meta-analysis. J Am Soc Nephrol. 2015;26(8):2055-2064. doi:10.1681/ASN.2014070688

15. Sumida K, Molnar MZ, Potukuchi PK, et al. Changes in albuminuria and subsequent risk of incident kidney disease. Clin J Am Soc Nephrol. 2017;12(12):1941-1949. doi:10.2215/CJN.02720317

16. Viazzi F, Ceriello A, Fioretto $P$, et al. Changes in albuminuria and renal outcome in patients with type 2 diabetes and hypertension: a real-life observational study. J Hypertens. 2018;36(8):1719-1728. doi:10.1097/HJH.0000000000001749

17. Schmieder RE, Schutte R, Schumacher H, et al. Mortality and morbidity in relation to changes in albuminuria, glucose status and systolic blood pressure: an analysis of the ONTARGET and TRANSCEND studies. Diabetologia. 2014;57(10):2019-2029. doi:10.1007/s00125-014-3330-9

18. Selvaraj S, Claggett B, Shah SJ, et al. Prognostic value of albuminuria and influence of spironolactone in heart failure with preserved ejection fraction. Circ Heart Fail. 2018;11(11):e005288. doi:10.1161/ CIRCHEARTFAILURE. 118.005288

19. Carrero JJ, Grams ME, Sang Y, et al. Albuminuria changes are associated with subsequent risk of end-stage renal disease and mortality. Kidney Int. 2017;91(1):244-251. doi:10.1016/j. kint.2016.09.037

20. Su JB, Zhao LH, Zhang XL, et al. HbA1c variability and diabetic peripheral neuropathy in type 2 diabetic patients. Cardiovasc Diabetol. 2018;17(1):47. doi:10.1186/s12933-018-0693-0

21. Khalil SA, Megallaa MH, Rohoma KH, et al. Prevalence of chronic diabetic complications in newly diagnosed versus known type 2 diabetic subjects in a sample of Alexandria population, Egypt. Curr Diabetes Rev. 2019;15(1):74-83. doi:10.2174/ 1573399814666180125100917

22. Tahrani AA, Dubb K, Raymond NT, et al. Cardiac autonomic neuropathy predicts renal function decline in patients with type 2 diabetes: a cohort study. Diabetologia. 2014;57(6):1249-1256. doi:10.1007/ s00125-014-3211-2 


\section{Publish your work in this journal}

Diabetes, Metabolic Syndrome and Obesity: Targets and Therapy is an international, peer-reviewed open-access journal committed to the rapid publication of the latest laboratory and clinical findings in the fields of diabetes, metabolic syndrome and obesity research. Original research, review, case reports, hypothesis formation, expert opinion and commentaries are all considered for publication. The manuscript management system is completely online and includes a very quick and fair peer-review system, which is all easy to use. Visit http://www.dovepress.com/testimonials.php to read real quotes from published authors.

Submit your manuscript here: https://www.dovepress.com/diabetes-metabolic-syndrome-and-obesity-targets-and-therapy-journal 